On January 10th the stupor had deepened somewhat. 'The temperature remained high in spite of frequent baths. During the day the infant had five convulsions. The convulsions were tonic and clonic, and lasted about one or two minutes. There was no rigidity of the head, or tenderness in the neck, or elsewhere. 'The extremities were lax. The abdomen was slightly distended but not tense. 'The child roused from lier stupor when handled. The pupils were equal and reacted to light. Nothing abnormal was detected in the heart or lungs. 'The ears were normal.

On January 11 th the general condition was worse. The stupor was more marked. The extremities were somewhat rigid. 'There was no tenderness in the neck or spine. The head was not retracted. There was no recurrence of the convulsions. The pupils were equal and reacted to light. 'The lungs were not abnormal. A loud blowing systolic murmur was heard over the whole cardiac area, especially loud over the mitral area. 'I'he heart's action was regular and very rapid. The respirations were from eighty to ninety in a minute and assumed a Cheyne-Stokes type at times. The infant appeared unable to swallow. During the last few hours the infant moaned with expiration. 'The ehild died quietly at 5.15 P. M., Jannary 11 th.

A utopsy (Dr. F. 13. Mallory). Heart. 'The valves and cavities are normal. 'The latter contain fluid blood.

Lungs. The lungs are downy to touch ; pink in color; there are no areas of atelectasis or of consolidation; there is a little serous fluid in the right pleural cavity.

Spleen. About normal in size; dark in color; on section the follicles are distinct; apparently no increase in pulp; the surface is moist.

Kidneys. 'The kidneys are injected; on section the surface is moist; the glomeruli show as red points; uric acid infaretion in the apiess of some of the pyramids.

Peritoneal cavity. The small intestines are distended with gas, with here and there contractions of the gut; a moderate quantity of thin, greenisls fluid in parts of the jejunum; a little semi-solid material in the cecum. The mesenteric lymph-glands are little if any enlarged. The lesser peritoneal cavity contains a small quantity of dirty brownish-black fluid surrounding the tail of the pancreas. No evidence of peritonitis is visible.

Esophaurus. Just above the cardiac orifioe on the right side the wall is softened in two longitudinal folds so as to be almost perforated; the adjoining mucous membrane is soft; there is no injection.

Stomach. One centimetre from the cardiac orifice, towards the fundus, there is a small perforation, three millimetres in diameter. 'The wall adjoining it is exceedingly thin and soft, consisting only of strunds of muscular tissue. The remainder of the stomach walls appear to be fairly normal. 'The gastric contents resemble the fluid found in the lesser peritoneal cavity.

'The intestinal mucous membrane is not abnormal.

Skull. 'The anterior fontanelle is open and large.

Brain. ()ver the convexities the meshes of the pia are distended and filled with a clear serous fluid, more or less separated into pockets by a yellow purulent infiltration especially of the upper layers of the pia. 'This infiltration extends in part diffusely throngh the pia but is especially noticeable accompanying each vessel of the pia in two parallel yellow lines, one on ench side of the vessel. 'The infiltration extends on each side to the bottom of the longitudinal fissure, and into the fissure of Sylvius. At the base of the brain the exudation seems rather more serous in character and extends down the cord. The bloodvessels of the pia and of the brain are moderately injected. 'The brain tissue is quite soft and on section transparent and moist. The lateral ventricles are somewhat dilated.

Bacteriological Examination. Cover-glass preparations from the sero-purulent exudation of the pia show enormous numbers of lancet-shaped diplococei, often in short chains of four to eight, surrounded by perfectly definite capsules.

Cultures. Spleen negative. Kidneys negative. Liver: one colony of diplococcus lanceolatus. Lungs: numerous pin-point colonies of micrococeus lanceolatus and a few colonies of streptococcus, many large colonies of staphylococcus aureus. Brain : two tubes, thirty-six hours after inoculation, show the surface of each tube covered with innumerable minute, transparent colonies; in one tube a single large white colony ; cover-glass preparations show lancet-shaped diplococei.

Anatomical Diagnosis. Acute cerebro-spinal meningitis due to the diplococcus lanceolatus; post-mortem softening of esopliagus and stomach.

Microscopic Examination (Dr. Wentworth). Portions of the brain were hardened in alcohol, mounted in parafin, and stained with hematoxylin and eosin. Additional sections were stained by Weigert's fibrin stain and by Gram's method.

There is a marked purulent infiltration of the pia-arachnoid. Most of the cells are polymorphonuclear leucocytes, but there is in addition a certain number of large cells with considerable protoplasm and large vesicular nuclei. 'These cells are probably derived from proliferation of the endothelial and fixed connective-tissue cells. A variable quantity of fibrin is found especially about the vessels and to some extent between the cells. The cells of the exudation are all well preserved and stain sharply. Sections stained by Gram's and Weigert's methods show typical pneumococci in small numbers scattered irregularly throughout the exudation. Along the surface of the cortex a slight invasion with leucocytes has taken place. 'The most marked lesion in the brain tissue, however, is found along the blood-vessels entering from the pia. The perivascular lymph-spaces are dilated and often contain an abundant growtl of pneumococci. In places leucocytes are found among the organisms, or in the tissue adjoining them. Occasionally small abseesses are present filled with more or less fragmented leucocytes, red blood globules and pneumococei; such foci have unquestionably arisen from extension of the organisms along the bloud-vessels and the production of foci of necrosis and infiltration. Many of the small arteries show slight thickening of the elastica and an increase of cells between it and the lining endothelium; a condition already described in Case $X$, where it was more marked.

I desire to thank Dr. L. M. Buckingham for his kiuduess in permitting me to use several cases of epidewic cerebro-spinal meningitis for purposes of illustration, and for the opportunities which 1 had for studying the disease in the wards of the Children's Hospital during the epidemic of last spring.

\section{THE PROPER'TIES OF SNAKE POISON.}

A Rrvikw of this Work of Fraser and Calmettre.

$$
\text { BY A. K. ATONK, M.1), }
$$

Physician to Out-Patients, Massachusetts General Hospital, Assistant in Bacteriology at the Harvard Medical sihool.

Inspikn by the results which have been obtained from the close study of bacteria and bacterial toxins, a number of observers have been led to turn their attention towards the interesting and fuscinuting subject of snake poisons. The similarity of their action to the bacterial poisons made it probable that similar methods of experimentation would give equally favorable results. Although the quantity of venom secreted by a given snake is much in excess of the amount to be obtained from many millions of bacteria, yet the difficulties aro in many ways greater than those which beset the bacteriologist. Tho latter has to do with short-lived plants which can for the most part be grown easily under artilicial conditions and reproduced at will for any number of generatious; while the 
difficulties in the way of collecting venomous snakes from the jungles of India, the banks of the Nile, from Australia and tropical America are ciear to all.

From a practical side the subject has a most important bearing. Such statistics as are at hand show that in British India alone about 20,000 persons lose their lives each year from the bites of suakes, and that the loss of cattle and other domestic animals is still more severe. Explorers and travellers in tropical regions are much annoyed, both by the loss of valuable camp-followers, pack animals or hunting dogs, as well as by the possibility of danger to themselves when going through the thick jungles. Furthermore, none of the cures for snake-bite have any very great value. Some of the observers and students upon the subject go so far as to say that alcohol, ammonia, strychuia, stimulants, and the diuretics and diaphoretics which produce rapid excretion through the skin and kidneys, have no effect whatever upon the action of the poison. Others are willing to allow that they all have some value, and that cases near the border-line of the minimal fatal dose are often saved by the action of these various remedies, but agree with the first-mentioned observers, that when the bite has been severe, and the dose hus been a large one, the drugs have very little or no effect upon the fatal course of the poison. Still a third class insists that they get sure results from the administration of various remedies. For example, strychuia in enormous doses is being recommended by Müller, of Australia, and many cases are quoted to prove the efficacy of this drug. The following is generally accepted, as the best treatment: If an arm or leg hus been bitten apply, first of all, a tourniquet, then wash the part at once with a solution of permanganate of potash or chromic acid, enlarging the wound to induce free bleeding, and aiding the removal of the poison by sucking; or freshly made chloride of lime (1-60) can be injected around the marks of the fangs. 'These measures, if undertaken early, are often effective in removing or oxidizing the venom so that lif $\theta$ is saved. The native doctor and snake-charmer often seem to have found some methods of successful treatment or of producing immunity. Too many wellauthenticated stories exist, showing immunity and cure, to make it at all doubtful that these persons huve methods which are doubtless of value. In all of these the head or poison glands or gall or blood of snakes plays a prominent part.

One thing which makes it difficult to estimate the value of uny given remedy is the impossibility of knowing how large a dose of venom has been introduced. When a suake strikes, if successful, ten to fifteen drops of the poison are usually discharged from the poison gland into the wound; or, as Calmette has found by experiment with snakes kept in confinement, that from 100 to 130 milligrammes of fluid poison, which would yield 20 to 50 milligrammes of dried poison, is discharged at a time. Whether this latter small amount is due to the fact that the snakes have been kept in confinement is not stated.

If a suake misses the first time it strikes the venom is nevertholess discharged; then on striking a second time there is much less poison in the gland which can be injected into the wound. Hence a person may be bitten by one of the mont venomous snakes and yet have only a few drops introduced, enough to produce very unpleasant symptoms, but not enough to cause death and consequently many cures may appear to be effected by various drugs which are in reality due to the small dose of the poison.

A question of much dispute has arisen, as to the difference of the various poisons. It has been generally believed that there were several distinct varieties of poison, and that snakes should be divided by the peculiarities of their poisons; but Calwette has asserted boldly in the last fow years that not only is the active principle of all snake poisons alike, but that it is identical in the poison of scorpions and lizards, and that the differences which have been noted in the action of the venom of various snakes was due to the presence of non-toxic albuminoid substances which are combined with the active poison in varying amounts. Thus the edema, the general hemorrhages and the hematuria which are observed for the most part as following wounds made by the less poisonous snakes are accounted for by the action of the practically inert albuminoids which can be removed by heat, leaving an active poison which can be dried and found to be of equal potency and similar action as similar products from the more poisonous suakes.

All the recent observers are agreed that an animal or person can be vaccinated against what would otherwise be a fatal dose by the subcutaneous introduction of small non-toxic doses of the poison. But the reasons therefore cause discussion. Fraser and Phisalix believe that antidotal properties reside in the venom along with the toxic. Fraser states his belief as follows: "that protection or immunity is chiefly due to the accumulation in the blood of an antidotal substance, which originates, at least in part, from the venom itself, and is normally one of the constituents of the venom." Calmette, on the other hand, denies this position entirely, believing that the antidotal properties are formed in the blood of the vaccinated animal by the organism itself. The experiments on this subject will be detailed later.

It has been found that the blood-serum of aniwals that had been vaccinated against snake venom had a certain autidotal power; though it was also observed that the blood-serum of several animals which were known to be immune to suake poison, the mongoose, for example, did not have antidotal properties. Fraser's experiments showed that the blood-serum of immunized animals gave him antitodal results and that the results were most marked when similar poison was employed and that in a less marked degree did the serum act to protect against the venom of other varieties of suakes. His method of experimentation was to mix the serum and the venom, having first determined what the minimal fatal dose might be, and then find out what was also the smallest ausount of serum necessary to neutralize this dose of venom. In vitro he was able to get regular results, but when he turned from this thoroughly artificial method to an attempt at a practical working he found that it took a great deal more serum to save life; even if the serum was introduced half an hour before the venom. Ho was inclined to believe that too exaggerated an idea of the valuo of the serum might gain ground. According to his calculations, it would take at least 300 c. c. or 10 or 11 oz. of the strongest rabbit serum that he was able to produce to save the life of un averagc-sized man who had been bitten by a venomous sorpent - an amount which would practically destroy the possibility of use. 
By further experiments he found that the bloodserum of venomous serpents and their gall possessed antidotul properties, but to a much less degree than the blood-serum of immunized animals.

Fraser next turned toward the ingestion of poison through the stomach for the production of immunity. That suake poison was inert when taken into the stomach had been known for a long time. Some of us will remember the impression made upon us by the description of making arrow poison in one of Captain Mayne Read's books, when, after adding the poison sacs of several snakes to the mixture, it was tasted to see if it had the proper consistency. Also the old method of sucking the poison from the wound had demonstrated often that the venom was harmless when taken into the stomach, and some suake-charmers say that they have acquired their immunity by this means. The poison is not absorbed by the walls of the stomach, nor is it changed by the action of the gastric juice, but in the intestine it is rapidly decomposed and rendered inert before it can be absorbed. 'This fact pointed to a possible antidotal action in the bile and to a later series of experiments.

Fraser's expériments were as follows: A cat was fed upon cobra poison for a month and at the end of that period taking more than eighty times the fatal dose, had the poison been introduced subcutaneously. The cat was not made sick by this diet, aud at the end of that period was found to be protected against one and one-half times the minimal fatal dose injected sub. cutaneously. White rats were fed $10,50,200,500$, 1,000 times the fatal dose of poison without harm. It was found that two hours were necessary to elapse in order to give protection and that when once acquired the animals would remain immune for five or six days. The results are of no practical value, as two hours is too long a time for the action of an antidote for such a rapidly working poison as snake venom.

From the consideration of the above facts and experiments, liraser concluded that the antidotal powers of the blood-serum were due to a chemical reaction, because, for example, a certain amount of serum mixed with five times the fatal dose could not neutralize the poison, if they were left in contact but ten minutes, but would if the process was allowed to continue for twenty minutes, that is, a definite time must elapse for a reaction to take place. 'The power of the serum is not destroyed by drying, neither is it injured, but is rather increased in power by bucterial action(?). Finally, immunity cannot be due to phagocytosis, this process does not exist in vitro but is a living process.

During the past year Fraser has worked on the antidotal properties of the bile. He reasoued that the bile was what was poured over the poison as it passed unchanged from the stomach, and consequently it was probably the neutralizer of the poison. Also it was well known that bile of venomous snakes formed an important part of the medicines of the India snakedoctors, some of them even refusing to aid in the collection of bile on the ground that the English would learn too much. It was found that in the same way as blood-serum of immunized animals, the bile of poisonous serpents mixed with the poison prevented lethal doses from producing death, and the amount needed is only slightly more than the blood-serum. 'The bile of innocuous snakes was also tried. Most of these have rudimentary poison-glands and they are immune against the bites of the venomous suakes, and their bile was found, as would be expected, anti-venomous, but to a less extent than the poisonous varieties. $O x$ bile was found to have neutralizing power, but only about one-seventh the strength of that of the venomous snakes; while the bile from rabbits and guinea-pigs was still feebler. But a decided obstacle to the use of bile as an antidote is the fact that bile itself is poisonous when introduced subcutaneously, and some of the animals experimented upon, though saved from the suake poison, died after four days, with symptoms of bile poisoning rather than suake poison.

An attempt was made to get at the active principle of the bile, and for this purpose bile of a puff-adder was treated with absolute alcohol, and after further treatment it was found that the portions of the poison soluble in alcohol had little anti-venomous properties. The portion of the bile insoluble in alcohol was treated with water and a dark green residue was left on evaporation, weighing about one twenty-sixth of the original bile. White rats were used for experiments; and when it had been determined that the amount of crude bile of the puff-adder sufficient to neutralize the minimum lethal dose of cobra poison was $.00025 \mathrm{mg}$. per kilo., the amount of the aqueous extract of the alcoholic precipitate necessary was but $.00001 \mathrm{mg}$. per kilo. 'The proportion here to the quantitative result is very striking. 'These results, it is to be borne in mind, were in vitro, and the quantity necessary to act on the living organism Fraser calculates as 1,600 to 2,000 times greater.

Calmette, who is the head of the Pasteur Institute at Lille, has been at work for a number of years upon the subject of toxins, and especially those of suake venom. In July of 1896 , before a committee of the Royal College of Physicians and Surgeons, of which Prof. Sims Woodhead was the chairman, Calmette made the following demonstration of the antidotal power of the serum of horses treated by repeated and increasing doses of snake venom:

(a) Six rabbits were taken and three cubic centimetres of the serum was introduced into the vein of the ear; five minutes later a dose of poison calculated to kill the animals in twenty minutes, if unprotected, was injected into these rabbits and also into two control animals. 'These latter died in sixteen and seventeen minutes respectively, while the six protected rubbits survived and remained well, though under observation for a number of months.

(b) Eight rabbits were taken, and each was injected with poison calculated to kill in about two hours time. One-half hour later three cubic centimetres of serum was introduced into the ear vein of two of them. At the end of an hour a similar amount was injected into a second pair, and at the end of un hour and a half a third pair were treated with five cubic centimetres of the protective serum. One of this last pair of rabbits died during the protective injection and the other about fifteen minutes later. 'The two control rabbits died in one hour and forty minutes, and one hour and forty-five minutes; those, however, that had been injected at the end of one-half and one hour lived.

(c) This experiment was to test the immunizing property of the serum. 'Three cubic centimetres were introduced intravenously, and fifteen minutes later a dose of poison which would be expected to kill the animal in twenty minutes was introduced and the animal lived.

(d) A dose of poison sufficient to kill in twenty 
minutes was introduced by the ear vein, and three minutes later three cubic centimetres of the protective serum was injected by the other ear vein; and the animal remained alive.

Convinced by these experimeuts, the committee recommended the use of the serum in India and Australia. And for more than a year the Institute at Lille has been sending out its serum to various parts of the world, and a number of favorable reports have begun to be received by Calmette, of which the following are exumples :

CAsE I was a boy working in the laboratory at Lille. He incautiously opened a box which contained a newly arrived lot of snakes and was promptly and severely bitten on the index finger. Almost immediatcly the hand and forearm became swollen aud edem. atous. An hour later there was insensibility of the forearm and hand, great pain in the upper arm and neck and axilla, and there was nausea. Twelve cubic centimetres were injected; and from that time the symptoms began to subside, and by the next day the sensibility in the hand had returned, the edema was less, and the boy was practically all right. A woman who chanced to be bitten by the same lot of snakes died in two hours, untreated.

Case II. A native camp-follower, a big man, was bitten in several places. One half-hour later he was seen, alarming symptoms having already set in. He was nearly comatose, with subnormal temperature and pulse of 140, which was weak and irregular. 'There was difficulty of respiration, and vomiting, spasin, pain in the legs and much edema. He was given an injection; the next morning was much better; and in two days returned to his work.

CAse III. A girl of thirteen was bitten by an Egyptian cobra, the asp of Cleopatra. 'Three or four hours later she was brought to the hospital in Cairo in a state of complete collapse, apparently a most desperate case. She was insensible; there were no reflexes, and the pupils were dilated and did not react to light. 'Twenty cubic centimetres of the serum were given at 7.30 , aud by 11 o'clock she seemed better and could answer questions; the pulse was 140. A second dose of 10 cubic centimetres was given, and within twelve hours she appeared to be out of danger.

So far reports have been received showing favorable results from similar cases that have been bitten by the following venomous snakes: (1) the naja-tripudians or cobra of Indo-China, (2) the bungarus cæruleus of India, (3) the naja-haje or usp of Egypt, (4) the najanoir of West Africa, (5) the bothrops lanceolatus of Ceutral America.

These favorable results lend further credence to Culmette's position that the active toxic property of all venoms is the same. 'To those who are interested in methods by which such serum is obtained and its strength determined, a brief statement of Calmette's methods may not be out of place. Firet of all he determines the dose of poison which is required to kill a rabbit in twenty minutes. Next he injects either just before or within ten minutes of the time the dose of poison is given one-half, one, or two cubic centimetres of the serum. If he finds, for example, that one cubic centimetre of serum is sufficient to save a rabbit weigh. ing two kilogrammes, he says that this serum contains 2,000 anti-venomous units. He makes and sells nothing less than serum containing 1,000 units, and for export to the countries where the most poisonous ser- pents are found he uses serum of the strength of 4,000 units. The dose therefore for a man of 75 kilogrammes is about 75 grammes or three ounces of the 1,000-unit serum. The serum appears to bear transportation and variations in temperature and to keep indefinitely in properly sealed bottles.

Incidentally, Calmette made a number of other interesting observations. He found, like Fraser, that his vaccinated rabbits remained immune for a considerable time; for example, one that had received finally a dose of 60 milligrammes of poison, or sufficient to cause death in 120 non-vaccinated rabbits, was able at the end of eight months to resist a dose which was capable of killing three rabbits. Also, animals which had become immunized rapidly lost their immunity within a few days. A female rabbit was found able to transmit her immunity providing the gestation took place during the height of the immunizing period, and the young retained this immunity for two months. On the other hand, an immunized male rabbit was unable to transmit his immunity to his offspring.

In attempting an analysis of the poison, Calmette, as has been said, agrees with certain observations made by various observers, but gives different interpretation to the facts. For example, he acknowledges that heat reduces the action of the poison. But he will not allow that there is any change brought about by the action of heat by which the poison is transposed into a vaccine, as Fraser and others have supposed, but that the toxic power is simply reduced and when injected into an auimal the result is simply that of introducing a small dose of normal virulent poison - antidotal properties developing in the serum of the organism. Phisalix, of Paris, in 1896 published some researches, conducted by passing venom through an unglazed porcelain filter. He found that the filtrate may be injected into rabbits in doses three or four times as great as the normal fatal amount, and the animals do not die, but are made immune. From this Phisalix concludes that he removes the active toxic principle by the aid of the filter, while the antitoxic material passes through the porcelain. Calmette maintains that this deduction is not warranted, and that the results obtuined are misleading. He makes a modification of the experiment as follows: A gramme of venom was taken, dissolved in water, heated to precipitate the albuminoids, and filtered to rewove them. The precipitate collected from the filter was found to be harmless and it was possible to inject the whole amount of albuminoid material without producing death. 'The filtrate, on the other hand, was strongly toxic, and the fatal dose cor. responded with the amount of the untrented poison. The filtrate was further dialyzed in order to remove all the salts. Tho residue was then evinporated to dryness over $\mathrm{H}_{2} \mathrm{SO}_{4}$, and he found that the material left was of great toxic power, as was shown by the fact that while the normal venom requires a dose of 0.6 of a milligramme to kill a 2,000-gramme rabbit in twenty minutes, the same result was brought about by a dose of this active toxic principle of .01 of a milligramme.

Comparing his results with those reported by Phisalix and confirmed by his own observation, he concludes that what takes place during the process of filtration is that the porcelain filter at first lets a small amount of the poison through its pores but quickly becomes coated by the albuminoid materials of the poison and as a result is transferred from a filter into a dia- 
lyzer and the toxic principle no longer passes through but only the salts of the poison; that the filtrate, so called, when injected, simply acts as a weak dose of poison.

'The filtrate obtained after precipitation by heat gives the biuret and Millon's reactions, but does not give the reaction of xantho-proteids. From such imperfect data it is impossible to state exactly what sort of an organic compound is present as the active principle of the suake poison.

From the above meagre report of the painstaking work that has been done upon this subject, it will be seen that decided steps in advance have been made in the past few years in our knowledge of the nature of snake poison, the most important of which is the assertion that all poisons are alike, and second, that serum from horses made immune to snake poison by the venom of the most poisonous snakes has the effect of counteracting the poison of all varieties of snake venom, and that the serum can be obtained of such strength and concentration as to make it a practical remedy. The short time, a year and a half, that this serum has been in general use makes it impossible to speak definitely about the matter, but the number of favorable cases already received makes it probable that a real antidote to suake poison has at last been found; though whether it can be produced in sufficient quantities to supply the demands of the tropics is not as yet demonstrated.

\section{Ulinical EDepartment.}

\section{GLIOMA OF 'THE BRAIN.'}

HY K. s. BOLAND, M.D.

J. W. P., forty-six yeurs of age, married, lad to give up work in September, 1896. He gave a history of fair general health, though never rugged physically and not very ambitious mentally, though ho had a fair education and when younger had preached some, and up to the time of his break-down he sung a correct and acceptable tenor in a church choir. For the past seven years, until September last, he had been employed as general utility man in one of our large manufucturing houses, and was a faithful and efficienc servant, well thought of by his employer. For some months before he broke down, his wife and daughters noticed that he was very forgetful, and also that he was more irritable though he would soon get over his annoyance and apologize if he, during the flurry, had spoken hastily. In addition, during most of the past seven months he complained more or less of head pains, which he located in the vertex and occipit. - He would wet his pillow with cold water before retiring, in the fancied belief that it afforded belief to the cephalalgia.

When I was called to hin it was near the end of September, 1896. He was sitting up, but complained of vertex pains and had a temperature of $101.5^{\circ}$, pulse 96. As there was anorexia and some conting of the tongue, I was on the lookout for typhoid. Under rest and antipyretics he improved so much that $I$ gave up the typhoid theory, and he went back to work after three or four days, but only for a day or two.

1 Reportod before tho Boston Medico-Psychologleal Society, Maro 18,1897 .
Any effort was too much for him, and extreme debility and his old head pains returned.

At this time, October 18, 1896, there was weak fast pulse, slightly elevated temperature, sluggish bowels, and much vertex heat and pain complained of. Mental state was almost normal ; no localized paralysis or impairment of special senses ; though his family thought his sight was not as good as formerly. 'There was more or less vomiting at this time. I inclined to the belief that he had cerebral meningitis, a view that Dr. C. F. Folsom, who saw him about the end of October, could not indorse.

He was admitted to the Boston City Hospital November 2d, where he remained eighteen days, admitted as suffering from hysteria, and discharged with diagnosis of cerebral tumor. No operation was advised fortunately, and he returned to his home about November 20th.

After his return from the hospital his symptoms grew rapidly worse, and he soon became totally blind and lost all sense of smell, though his hearing remained first-rate. His stomach grew more unstable, less and less food was retained, and morphia had to be given to relieve the cephalalgia and wakefulness.

About January lat he was found with the left side totally paralyzed; there was incontinence of urine and feces, and no perception of light or odors. Bed-sores developed, and though still able to speak, he sank fast, and died January $26,1897$.

At the autopsy a clear cyst, as large as a dried date, lay on the floor of the right lateral ventricle just above the corpus striatum, whose suriace, however, it did not depress or in any way give evidence of injurious pressure to the surrounding parts.

The left occipital lobe was occupied by a new growth that at the posterior extremity was so firmly adherent to the dura that it was somewhat torn and on removal the dura had to be removed with it.

Dr. W. F. Whitney's appended report will best describe it :

An illy-defined mass, replacing and infiltrating the posterior part of the occipital lobe for a distance of about ten centimetres in length and the entire breadtls. Its anterior edge was about the line of the parieto-occipital fissure. The outer surface was irregular and ragged, and at the posterior part was adherent to the dura mater. The section surface showed a soft, grayish mass, dotted with dark red (hemorrhagic) spots, and yellow opaque (necrotic) areas. ('l'he brain was torn and lacerated when received, probably during removal, from the very soft character of the growth, so that a more exact localization was impossible.)

Microscopic Examination. - The structure was found to be a growth of small cells of irregular shape, with very fine prolongations associated with them, and which apparently passed between them, giving to the whole an aspect of very closely felted cells and fibres. An occasional ganglion cell could be found in the mass, and the transition into the intact brain tissue was perfectly gradual.

Diagnosis. - Small-cell glioma of the right occipital lobe.

I report this case for information. Why was this man blind and why did he lose the sense of smell while hearing was intact? Is it usual to find in cerebral pathology as large an amount of brain tissue involved and otill giving 80 little evidence until so late in the disease?

'Tus number of cases of, and deaths from, typhoid fever in Philadelphia greatly decreased last week. 